\title{
HPV Genotypes distribution in Indian women with and without cervical carcinoma: Implication for HPV vaccination program in Odisha, Eastern India
}

\author{
Rashmirani Senapati ${ }^{1}$, Bhagyalaxmi Nayak², Shantanu Kumar Kar ${ }^{3}$ and Bhagirathi Dwibedi ${ }^{*}$
}

\begin{abstract}
Background: Considering the limited cross protection offered by the current HPV vaccines, understanding the HPV genotype distribution among the different population is essential in predicting the efficacy of current vaccine and devising new vaccine strategy. The present work aimed at investigating the HPV genotypes distribution among women with and without cervical carcinoma in Odisha, Eastern India.

Methods: A total of 607 participants have been enrolled between January 2014 and June 2016. L1-PCR, sequencing, and E6/E7 nested multiplex type- specific PCR were performed for HPV detection and genotyping. Cytological distribution of 440 cases includes invasive cervical carcinoma or ICC $(n=210)$, inflammatory smear $(n=162)$, normal cytology $(n=68)$. Statistical analyses were performed by using SPSS version 20.0 software and MediCal version 14.10.2(7). A $p$-value of $\leq 0.05$ was considered statistically significant.

Results: The overall prevalence of HPV infection was (359/595) 60.33\%. Prevalence of HPV infection was 93. 80\% (197/210) in invasive cervical cancer (ICC) cases, 54.32\% (88/162) in inflammatory smear and 19.11\% (13/ 68) in normal cervical cytology. The most prevalent genotype was HPV16 (87.28\%) followed by HPV18 (24. 56\%) and HPV 51(3.46\%). The overall prevalence of single type was $76.58 \%$ and highest (78.9\%) among ICC cases. The most frequent genotype combination after HPV16 + 18(9.4\%) was HPV16+66+68(2.7\%) which was frequently observed in inflammatory cytology. Age $>45 y e a r s$, parity $\geq 3$, low socio-economic condition, rural residential area and post menopause state were significantly associated with HPV infection. Multiple infections did not have a significant association with any of the clinicopathological variables (stage, LN metastasis, cell type) except tumor size $\geq 2 \mathrm{~cm}$ in ICC cases. The impact of $2 \mathrm{~V}, 4 \mathrm{~V}$, and $9 \mathrm{v}$ vaccines in preventing cervical cancer in Odisha were $89.99,91.65$, and $92.16 \%$ respectively.

Conclusion: This data would help planning an appropriate strategy for disease monitoring and provides baseline data for post-vaccination surveillance in the region. The nonavalent vaccine would be significant in preventing cervical carcinoma in Odisha. Hence an effective vaccination program based on regional HPV epidemiological profile along with the cervical cancer screening is necessary to reduce the cervical cancer burden in India.
\end{abstract}

Keywords: HPV genotype distribution, Cervical cancer, Impact of HPV vaccine, Odisha, Eastern India

\footnotetext{
* Correspondence: bhagirathidwibedi@yahoo.com

${ }^{1}$ Viral Research and Diagnostic Laboratory, Regional Medical research Centre

(ICMR), Nalcosquare, Chandrasekharpur, Bhubanewar 751023, Odisha, India

Full list of author information is available at the end of the article
}

(c) The Author(s). 2017 Open Access This article is distributed under the terms of the Creative Commons Attribution 4.0 International License (http://creativecommons.org/licenses/by/4.0/), which permits unrestricted use, distribution, and reproduction in any medium, provided you give appropriate credit to the original author(s) and the source, provide a link to the Creative Commons license, and indicate if changes were made. The Creative Commons Public Domain Dedication waiver (http://creativecommons.org/publicdomain/zero/1.0/) applies to the data made available in this article, unless otherwise stated. 


\section{Background}

Cervical cancer is the fourth most common cancer in women and seventh most common cancer among all the known group of cancers found worldwide [1]. 528,000 new cases of cervical cancer recorded in the year 2012 [1]. Death due to cervical cancer estimated to be 266,000 worldwide. It accounts for $7.5 \%$ of all female cancer deaths [1]. 123,000 new cases of cervical cancer with 67,000 deaths recorded in India in the year 2012 [1].

HPV infection plays a central role in causing cervical cancer. Among 184 different HPV genotypes, only 40 diverse types can infect anogenital region which can be classified into 3 classes based on their oncogenic potential. HPV16, 18, 31, 33, 35, 39, 45, 51, 52, 56, 58, 59, 68, 73 and 82 are included in high-risk group while HPV6, 11, 40, 42, $43,44,54,61,70,72$ and 81 are included in low-risk group whereas HPV 26, 53 and 66 belong to the group of intermediate risk $[2,3]$. Considering the limited cross protection offered by the current vaccines, understanding the genotype distribution among different population is essential in predicting the efficacy of current vaccine and devising new vaccine strategy [4]. Epidemiology of HPV infection and pattern of HPV genotype distribution are not well documented in the entire Indian subcontinent which limits the implementation of cervical cancer prevention programs. Available reports from studies covering few parts of India show a wide variation in the prevalence of HPV infection and genotypes distribution which is attributed to diversified socio-economic and geo-climatic condition [5-9]. This necessitates a similar evaluation of the same in different geographical region of the country.

Here we determined the genotypes, prevalence and associated risk factors among women in Odisha with and without cervical cancer.

\section{Methods}

\section{Study population and sample collection}

Acharya Hari Hara Regional Cancer Center and SCB medical college, Cuttack, Odisha, the two apex referral hospitals of the state were considered to enroll subjects and cervical sample collection. This study was conducted between January 2014 and June 2016. Married women, above 18 years showing any of the symptoms like abnormal vaginal bleeding/discharge, pain during coitus, lower abdominal pain and clinician suspicion of cervical malignancy were included in the study after clinical examination by a gynecologist. Unmarried women, pregnant cases and patients undergoing treatment were excluded. Subjects were enrolled after getting informed written consent from them. This study is approved by the ethics committee of Regional medical research center (ICMR), Bhubaneswar, Odisha, India.

Clinical data including signs and symptoms and sociodemographic data such as age, education, economic status, age at marriage, the age of menopause were collected by interviewing the patients with a predesigned questionnaire.

Cervical swab specimen was collected using cytobrush and stored inside the viral transport media (Hi-Media) and transported to Virology laboratory, Regional medical research center, Bhubaneswar, Odisha for further analysis. Pap smears were prepared from the collected cervical sample for cytological analysis. Cytological classification was done according to Bethesda system [10]. Part of the tissue biopsy samples which was taken for diagnostic and patient management purpose were collected from confirmed cases of cervical carcinoma for histopathological analysis.

\section{DNA extraction and HPV DNA detection}

DNA was extracted from a $200 \mu$ l aliquot of exfoliated suspended cell samples using the QIA amp DNA Blood Mini Kit (Qiagen) as per the manufacturer's instructions. Amplification of the human $\beta$-globin gene was performed to test sample sufficiency. To detect HPV, PCR was performed by using PGMY09/PGMY11 primers (MY11: 50-GCMCAGGGWCATAAYAATGG-30; MY09: 50-CG TCCMARRGGAWACTGATC-30-) targeting a 450-bp region of the HPV L1 gene.

\section{Genotyping by sequencing}

L1 PCR products were isolated from agarose gel and purified. Sequencing was done using Big Dye Terminator sequencing kit (Applied Biosystems, Foster City, CA, USA) [11] and analyzed in silico on ABI 3100 Genetic Analyzer (Applied Biosystem). The results were then compared with the sequences available in the Genebank database using the National Center for Biotechnology Information BLAST program (http://www.ncbi.nlm.nih.gov/BLAST/).

\section{Genotyping of HPV by nested type specific multiplex E6/E7 PCR}

PCR followed by sequencing didn't resolve multiple infections. Hence, E6/E7 type-specific PCRs were performed to confirm multiple types. Type-specific primers were used to detect $18 \mathrm{HPV}$ genotypes that include HPV16, HPV18, HPV31, HPV 33, HPV 35, HPV39, HPV 56, HPV 59, HPV 45, HPV 51, HPV 52, HPV 58, HPV 66, and HPV 68, HPV 6/11, HPV 42, HPV 43, and HPV 44 genotypes. Primers were obtained by following the available literature [12]. The whole process includes two PCR reaction; the first PCR using E6/E7 primers followed by the type-specific nested PCR.

Briefly,50 $\mu \mathrm{l}$ PCR reaction mixture containing, 10X Taq buffer with Mgcl2 (Biotools, Spain), 250mM dNTP (Biotools, Spain), 5U Taq DNA polymerase (Biotools, Spain), 20 pmol of each primer and 50ng of DNA was processed for the first PCR cycle. The cycling parameters were composed of $4 \mathrm{~min}$ of an initial denaturation 
step at $94{ }^{\circ} \mathrm{C}$, followed by 35 amplification cycle. Each cycle includes the 30s of denaturation at $94{ }^{\circ} \mathrm{C}$, 30s of annealing at $56{ }^{\circ} \mathrm{C}$, elongation of $72{ }^{\circ} \mathrm{C}$ for $45 \mathrm{~s}$ and a final extension of $4 \mathrm{~min}$ at $72{ }^{\circ} \mathrm{C}$. Nested PCR reaction was same as the first PCR except for primers and sample. $2 \mu \mathrm{l}$ of the PCR product was used for the nested PCRs. 10 $\mu \mathrm{l}$ of the amplification products was analyzed by electrophoresis on $2 \%$ agarose gels stained with ethidium bromide followed by visualizing under UV light.

\section{Statistical analysis}

Multivariate logistic regression analysis was performed to determine the risk factors associated with HPV infection. Bivariate analysis was done to determine the variables associated with multiple infections in cervical cancer cases. Statistical analyses were performed by using SPSS version 20.0 software and MediCal version 14.10.2 [13]. A $p$-value of $\leq 0.05$ was considered as statistically significant.

Low and a high estimate of vaccine impact were Calculated by using the following formula:

For the quadrivalent vaccine, low estimate was the prevalence of HPV 6/11/16/18 genotypes alone or in association but excluding presence of another HPV type; high estimate was the prevalence of HPV 6/11/16/18 genotypes alone or in association possibly in presence of another HPV type. For the nonavalent vaccine, low estimate was the prevalence of HPV 6/11/16/18/31/33/45/52/58 genotypes alone or in association but excluding presence of another HPV type; high estimate was the prevalence of
HPV 6/11/16/18/31/33/45/52/58 genotypes alone or in association possibly in presence of another HPV type [14]. To estimate the impact of respective vaccine, the average of the low and high estimate was considered.

Proportion of additional cases potentially prevented by one vaccine compared to the other vaccine was calculated as per the previously published study [14].

\section{Result}

Enrollment of cases and outcomes were presented in Fig. 1. A total of 607 participants were being enrolled in the study. Among all the enrolled cases 12 cases were excluded from further analysis as they were negative for beta globin PCR. Cytological information was known for 440 cases only which includes normal $(n=68)$, cervicities $(n=162)$, invasive carcinoma $(n=210)$.

\section{Demographic and clinical features of cases}

Socio-demographic features of 595 subjects are presented in Table 1 . Mean and median age of the enrolled cases were $48.47(\mathrm{SD}=12.47)$ and 48.5 years respectively with an age range of 19-86 years. The mean age of marriage was $19.97(\mathrm{SD}=3.98)$ years with an age range of 14-37 years. 401 cases were with parity $\leq 3$ and 255 cases were in the postmenopausal state (Table 1). A majority of the cases $(83.52 \%)$ were illiterate/just literate and belongs to low socio-economic class (60.33\%).

The common clinical features recorded were abnormal discharge with or without blood stain (55\%),

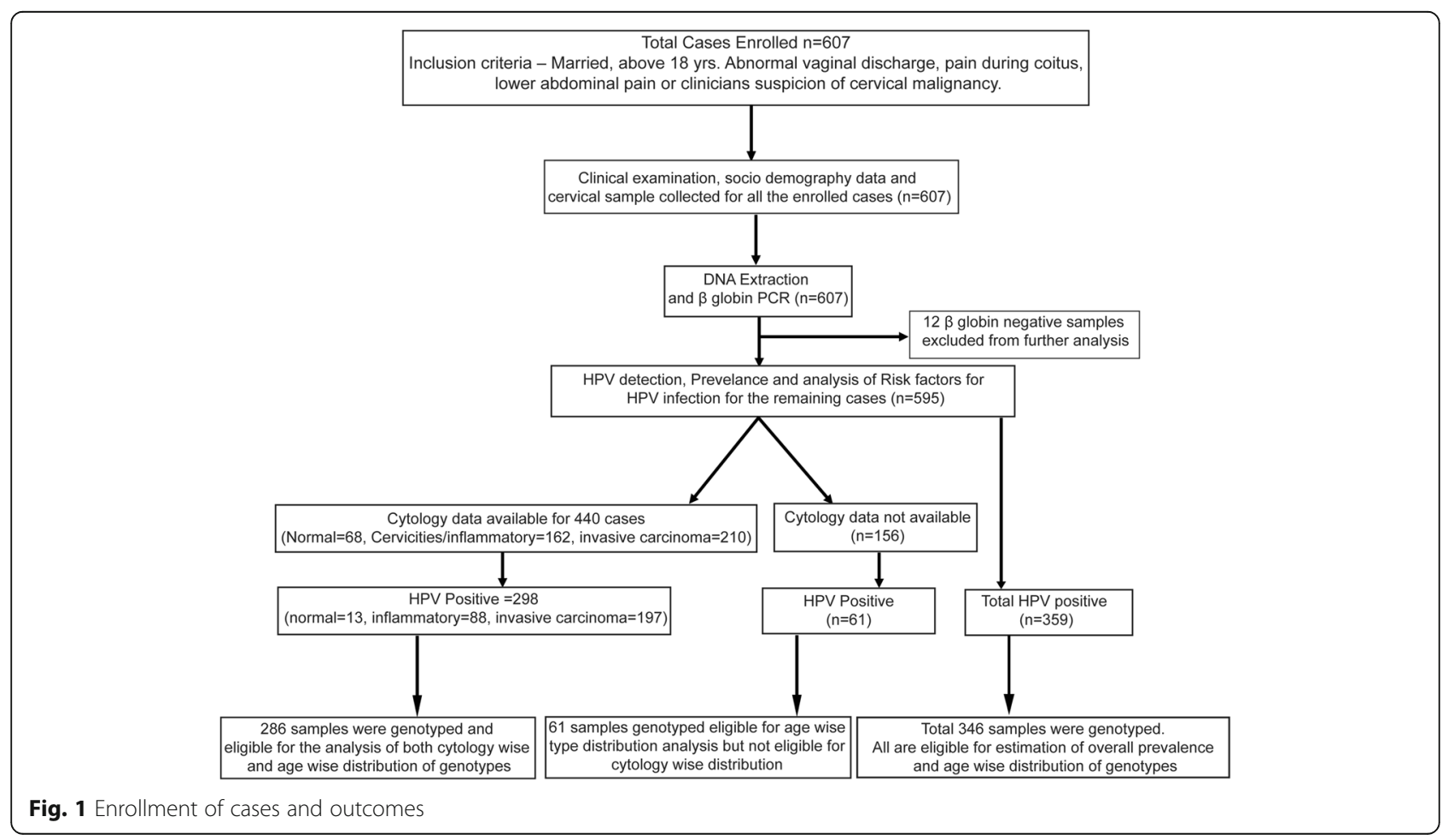


Table 1 Sociodemographic and personal characteristics of the population $(n=595)$ as risk factors for HPV infection ( $n=595)$

\begin{tabular}{|c|c|c|c|c|}
\hline Factors & HPV + ve $n=359$ & HPV -ve $n=236$ & OR $(95 \% \mathrm{Cl})$ & $P$ \\
\hline Age $>45 N=369$ & 236 & 133 & $1.4(1.06-2.08)$ & .02 \\
\hline Age $\leq 45 n=226$ & 123 & 103 & & \\
\hline Parity $>3 n=194$ & 136 & 58 & $1.87(1.29-2.69)$ & .0008 \\
\hline Parity $\leq 3 n=401$ & 223 & 178 & & \\
\hline Contraceptive Yes $n=557$ & 335 & 222 & $0.88(.44-1.73)$ & .71 \\
\hline No $n=38$ & 24 & 14 & & \\
\hline Age of marriage $\leq 18 n=234$ & 153 & 81 & $1.42(1.01-1.99)$ & .043 \\
\hline Age of marriage $>18 n=361$ & 206 & 155 & & \\
\hline Tobacco/betel Yes $n=289$ & 179 & 110 & $1.13(0.81-1.58)$ & .43 \\
\hline No $n=306$ & 180 & 126 & & \\
\hline Education No $n=497$ & 304 & 193 & $1.18(0.76-1.83)$ & 0.4567 \\
\hline Yes $n=98$ & 56 & 42 & & \\
\hline Low socioeconomic condition $n=359$ & 245 & 114 & $1.62(1.13-2.31)$ & 0.0081 \\
\hline High socioeconomic condition $n=200$ & 114 & 86 & & \\
\hline Rural $n=361$ & 322 & 39 & $20.97(12.65-34.76)$ & $<0.0001$ \\
\hline Urban $n=131$ & 37 & 94 & & \\
\hline Poor Menstrual hygiene $n=492$ & 306 & 186 & $1.55(1.01-2 . .37)$ & 0.0437 \\
\hline Good Menstrual hygiene $n=103$ & 53 & 50 & & \\
\hline Post menopause $n=255$ & 170 & 97 & 1.85 (1.33 to 2.57$)$ & 0.0002 \\
\hline Pre menopause $n=340$ & 165 & 175 & & \\
\hline
\end{tabular}

postmenopausal bleeding (20\%), bleeding and pain during coitus $(2 \%)$, lower abdominal pain $(17 \%)$, intermenstrual bleeding (14\%), prolapse (6\%), and swelling abdomen (1\%).

\section{Clinicopathological characteristics of ICC cases}

As per the International Federation of Gynecology and Obstetrics (FIGO), ICC cases were classified into different stages which include IB $(n=9)$, IIA $(n=8)$, IIB $(=38)$, IIIB $(n=111)$, IIIA $(n=2)$ and IVA $(n=4)$. The histopathological result of 172 cases showed that 167 cases belonged to Squamous cell carcinoma (SCC) and only 5 cases identified with Adenocarcinoma (ADC). Based on the available data for 89 cases, only 30 cases had tumor size $\geq 2 \mathrm{~cm}$ and 59 cases had $<2 \mathrm{~cm}$. Similarly, out of 86 available reports 30 cases had lymph node metastasis whereas 56 cases didn't have lymph node metastasis (Additional file 1: Table S5).

\section{Prevalence and analysis of risk factors for HPV infection} The overall prevalence of HPV infection was found to be (359/595) 60.33\%. Prevalence of HPV infection was $93.80 \%(197 / 210)$ in invasive cervical cancer (ICC) cases, $54.3 \%(88 / 162)$ in inflammatory smear and $19.11 \%(13 /$ 68 ) in normal cases (Fig. 1). Risk factors for HPV infection were analyzed by multivariate logistic regression analysis (Table 1). Age group $>45$ years, parity $\geq 3$, low socio-economic condition, rural residential and postmenopausal states were found to be significantly associated with HPV infection.

\section{HPV genotype distribution}

Three hundred forty-six samples were processed for genotyping by type-specific nested multiplex PCR. The most commonly detected genotype was HPV16 (87.28\%) followed by HPV18 (24.56\%) (Additional file 1: Table S1). Other detected genotypes in descending order were HPV 51(3.46\%), HPV 39(3.17\%), HPV 66(2.8\%), HPV 68(2.3\%), HPV 35(1.7\%), HPV 45(1.7\%), HPV 44(1.1\%), HPV 58 (1.1\%), HPV 52(.57\%), HPV 6/11(.57\%), HPV 42(1.1\%) and HPV 43(.57\%) (Additional file 1: Table S1). Prevalence of single and multiple genotypes was $76.58 \%$ and $23.41 \%$ respectively (Additional file 1: Table $\mathrm{S} 1$ ).

Cytology-wise HPV genotype distribution was done in 286 samples which includes normal cytology $(n=13)$, inflammatory smear $(n=88)$ and ICC $(n=185)$ (Additional file 1: Table S2). Among the women with normal cytology, 53.84\% were infected with single genotype of HPV16 while rests were infected with coinfection of HPV16 and 18. No other genotypes were observed in this group (Additional file 1: Table S2).

Among the women with inflammatory cytology HPV $16(89.77 \%)$ was the most predominant genotype followed 
by HPV $18(28.4 \%)$. Other genotypes detected among this group were HPV 66(6.8\%), HPV 68(6.8\%), HPV51 (3.4\%) and HPV35 (2.27\%) (Additional file 1: Table S3). In this group $70.45 \%$ cases were infected with single genotypes while $29.54 \%$ cases were infected with multiple HPV types (Additional file 1: Table S2). Infections with double genotypes found in $19.31 \%$ of cases while $10.22 \%$ cases were infected with triple genotypes combinations (Additional file 1: Table S2). The most common combination of coinfection in this group was HPV $16+18$ (14.72\%) followed by HPV16 + 66 +68(6.81\%) (Additional file 1: Table S2).

Among invasive cancer cases the most prevalent genotype was HPV16 (83.78\%) followed by HPV 18(21.08\%) and HPV 51(5.4\%) (Additional file 1: Table S3). Other genotypes in invasive cancer were HPV 45, HPV35, HPV66, HPV68, HPV44, HPV43, HPV42, HPV58 and HPV 52(Additional file 1: Table S3). Among the ICC cases $78.9 \%$ cases were infected with single genotypes while $21.08 \%$ cases were with multiple genotypes including double (11.89\%), triple (5.94\%) and quadruple (3.24\%) combinations of genotypes (Additional file 1: Table S2). The most prevalent genotype combination as coinfection among ICC cases was HPV16 + 18(4.32\%) followed by HPV16 + 39(2.16\%) (Additional file 1: Table S2).

Single genotype increases with the severity of the lesion. Single HPV16 also increases with the disease severity and highest in cervical carcinoma cases (Additional file 1: Table S2). Prevalence of HPV16 + 18 decreases with lesion severity and found to be lowest in ICC cases (Additional file 1: Table S2). The number of genotypes increases with the grade of the lesion (Additional file 1: Table S2).

Distribution of high-risk and low-risk HPV genotypes in the study population has been summarized in Additional file 1: Table S3. No low-risk genotypes were detected among the cases with normal and inflammatory cytology. All the cases with inflammatory cytology were infected with high risk genotypes except 6 cases which were infected with HPV66, an intermediate risk genotype. High risk genotypes were detected in all the ICC cases. Besides, low-risk (6.4\%) and intermediate-risk genotype $(2.16 \%)$ were also detected in ICC cases but in association with the high-risk genotypes. The low risk genotypes detected were HPV6/11, HPV44, HPV43 and HPV42 while HPV 66 was the only intermediate risk genotype.

\section{Prevalence and distribution of HPV genotypes infection in different age group}

Irrespective of cytology, the overall prevalence of HPV infection was highest (71.25\%) among women aged $>55$ years (Additional file 1: Table S4). The age wise HPV infection curve for the cases without cancer depicted a bimodal peak as shown in Fig. 2. It shows two peaks, one at the age of $\leq 35$ years with highest prevalence and another at $>55$ years. Figure 3 shows the age wise prevalence of multiple infections in women with and without cancer. Among the women without cancer the prevalence of multiple genotypes was highest in women aged between 36 and 45 years while in ICC cases it is highest among women aged $>55$ years. Among ICC cases the curve was bimodal showing two peaks, one at the age of 36-45 years and another at the age of $>55$ years. But in normal cases only one peak at the age of 36-45 years was observed.

\section{Impact of $2 v, 4 v$ and $9 v$ vaccine in preventing cervical carcinoma in Odisha}

Low estimates of genotypes targeted by $2 \mathrm{v}, 4 \mathrm{v}$, and $9 \mathrm{v}$ vaccine were $152(82.16 \%), 152(82.6 \%)$ and156 (84.32\%) respectively. High estimates of genotypes targeted by $2 \mathrm{v}$, $4 \mathrm{v}$, and $9 \mathrm{v}$ vaccines were 181(97.83\%), 183(98.91\%) and $185(100 \%)$ respectively. Absolute impact of $2 \mathrm{v}, 4 \mathrm{v}$ and $9 \mathrm{v}$ were $89.99,91.65$ and $92.16 \%$ respectively. The additional impact of $9 \mathrm{v}$ was increased when compared with $4 \mathrm{v}$ and $2 \mathrm{v}$ (Table 2).

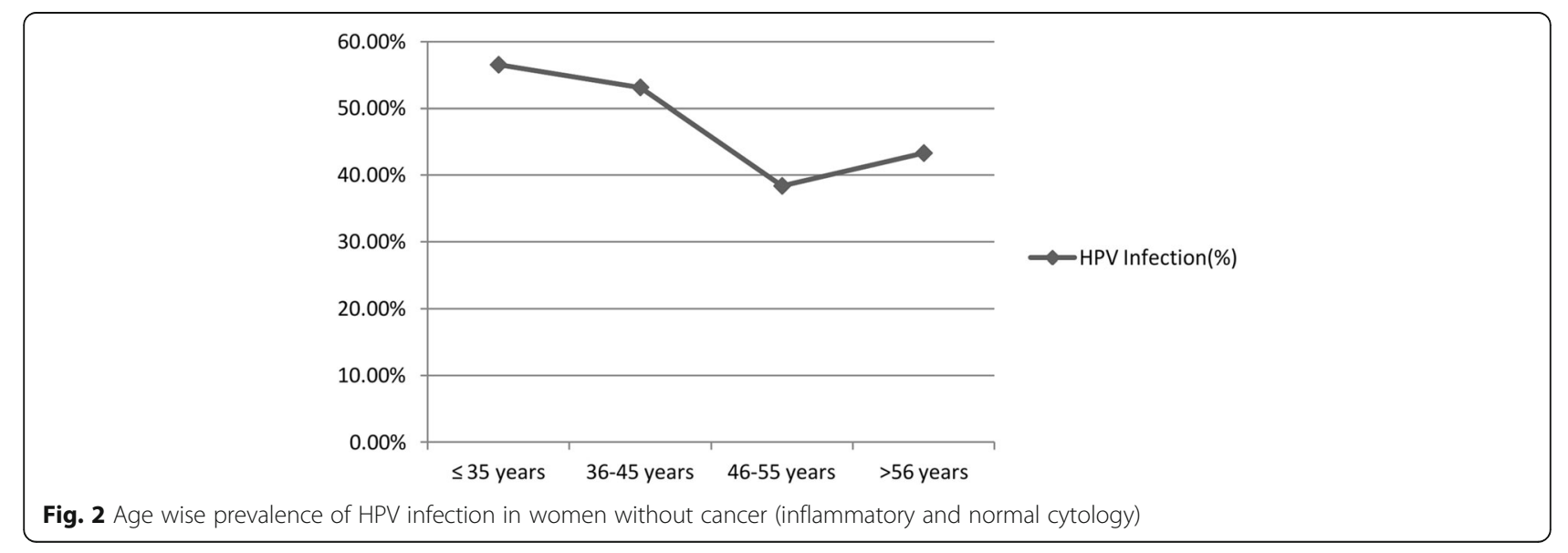




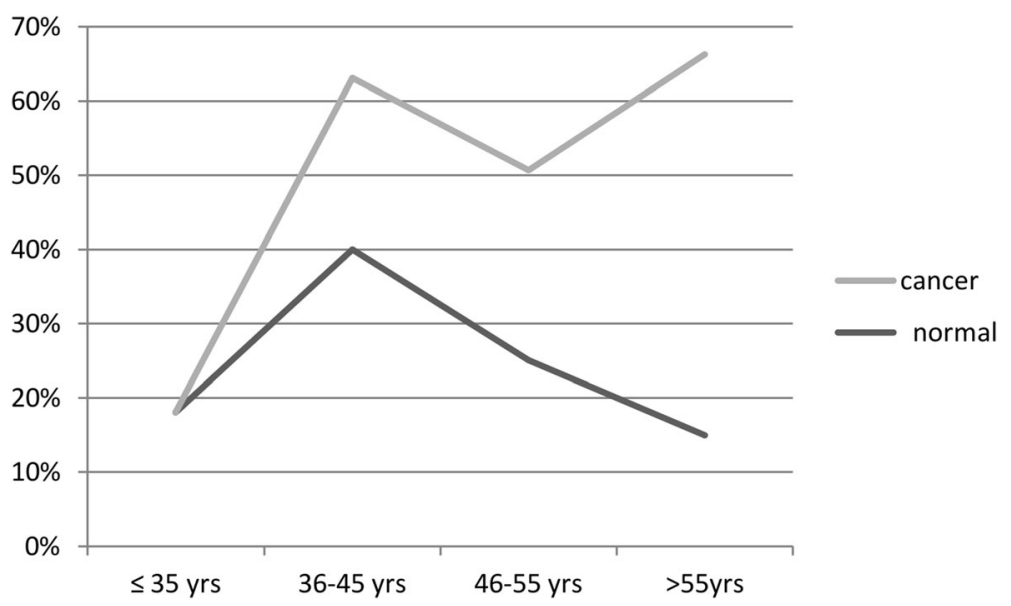

Fig. 3 Trends of Multiple infections among different age group in normal and cervical cancer

\section{Association between clinicopathological variables with multiple genotypes}

Association of clinicopathological variables with multiple genotypes have been shown in Additional file 1: Table S5. Among the ICC cases, the proportion of multiple types was more common in patients with aged $>50$ years as compared with aged $\leq 50$ years. The frequency of multiple genotypes was higher among patients diagnosed at a late stage than those at an early stage. Frequency of multiple infections was also higher in the cases with lymphnode metastasis than the cases without LN metastasis. No significant association was found between multiple genotypes and clinicopathological variables such as age at diagnosis ( $\leq 50$ years $v s>50$ years), stage at diagnosis (early stage or stage I-II $v s$ late stage or stage III-IV), lymph node metastasis (yes $v s$ no) and histopathology (Squamous cell carcinoma vs Adenocarcinoma). Multiple types were found to be significantly associated with tumor size $\geq 2 \mathrm{~cm}$ (OR $=$ $4.9595 \% \mathrm{CI}=1.68-14.51 p=.0035$ ) (Additional file 1: Table S5).

\section{Discussion}

The prevalence of cancer cervix is high in India [1] but countrywide data on HPV infection and genotype distribution is not available which would have been useful for a wider vaccination program. To the best of our knowledge, the current study is the first to report the prevalence of HPV infection and genotype distribution among the women with cervical diseases in the state of Odisha. Odisha is an eastern state of the Indian subcontinent covering an area of $1155,820 \mathrm{~km}$ with 45 million populations, known for her socio-economic backwardness and various public health issues. Samples were obtained from two apex referral hospital to which patients from all the districts of Odisha visit for consultation. Therefore, the study provides a precise estimation of the HPV prevalence and genotype distribution in symptomatic women of the state.

Compared to other studies in India, the present study disclosed a high prevalence of HPV infection among the women with normal cytology showing minor gynecological complaint [5, 6, 15-17]. This study, however, did not look

Table 2 Impact of $2 v, 4 v$ and $9 v$ vaccine based on the ICC cases infected with vaccine targeted genotype

\begin{tabular}{|c|c|c|c|}
\hline & Lowest estimation & Highest estimation & $(\mathrm{LE}+\mathrm{HE} / 2) \%$ \\
\hline Cases infected with genotypes targeted by $2 \vee$ vaccine(HPV16/18) & $152(82.16 \%)$ & $181(97.83 \%)$ & $89.99 \%$ \\
\hline Cases infected with genotypes targeted by $4 \mathrm{v}$ vaccine(HPV16/18/6/11) & $152(82.6 \%)$ & $183(98.91 \%)$ & $91.65 \%$ \\
\hline Cases infected with genotypes targeted by $9 v$ vaccine(HPV16/18/6/11/31/33/45/52/58) & $156(84.32 \%)$ & $185(100 \%)$ & $92.16 \%$ \\
\hline Absolute additional impact of $4 \mathrm{v}$ vaccine (compared with $2 \mathrm{v}$ vaccine) & - & $1.08 \%$ & $1.08 \%$ \\
\hline Relative additional impact of $4 \mathrm{v}$ vaccine (compared with $2 \mathrm{v}$ vaccine) & - & $1.09 \%$ & $1.09 \%$ \\
\hline Absolute additional impact of $9 v$ vaccine(compared with $2 v$ vaccine) & $2.1 \%$ & $2.16 \%$ & $2.16 \%$ \\
\hline Relative additional impact of 9v (compared with $2 \mathrm{v}$ vaccine) & $2.4 \%$ & $2.16 \%$ & $2.28 \%$ \\
\hline Absolute additional impact of $9 \mathrm{v}$ vaccine (compared with $4 \mathrm{v}$ vaccine) & $2.1 \%$ & $2.16 \%$ & $2.16 \%$ \\
\hline Relative additional impact of $9 \mathrm{v}$ vaccine (compared with $4 \mathrm{v}$ vaccine) & $1.08 \%$ & $1.09 \%$ & $1.085 \%$ \\
\hline
\end{tabular}


for any bacterial, fungal or HIV infection which could make them prone to HPV infection in symptomatic cases.

Prevalence of HPV among the cervical cancer cases was $94.28 \%$ in the present study. It is generally accepted that HPV virtually causes $100 \%$ of cervical carcinoma. Hence, the difference in results could be partly explained by differences in the sensitivity of the HPV detection techniques used. The most prevalent genotype in cervical cancer was HPV 16 followed by18 is in accordance with international and local data $[7,8,16]$. As compared to other studies, the overall prevalence of HPV 16 and 18 in cancer cases $(82.3 \%)$ is much higher in the present work $[7,8,16]$.

Analysis of genotypes distribution in ICC cases showed that HPV 16(83.78\%) and 18(21.08\%) were the most predominant genotypes which is quite similar to the studies reported from India and worldwide. In Kolkata, $59-74 \%$ of ICC cases are infected with HPV 16 and $2-13.9 \%$ cases infected with HPV 18 [7, 18]. Reports from south India showed HPV 16 and HPV18 accounts for $58-69 \%$ and $5-19.4 \%$ of ICC cases respectively $[16,18]$. In a similar study from Delhi (north India) reported that HPV 16 and 18 contributing 73.6 and $14.2 \%$ cases of cervical carcinoma [19]. Other parts of Central and west India also have similar reports showing HPV 16(72-73.6\%) as the most predominant genotype followed by HPV18 (5-11.9\%) in cervical carcinoma cases [18, 20]. In Pakistan, HPV 16 and 18 accounts for 45.1$94.9 \%$ and $1.7-43.1 \%$ of ICC cases respectively [2124]. However, in the Chinese population, HPV 16 is the most predominant followed by HPV 52 rather than 18 [25]. Distribution of most common genotypes in ICC cases is also consistent with the types found in worldwide [26, 27].

HPV 51 was found to be the 3rd most predominant genotype in the present study, though it is rarely reported in other regions of the country and worldwide. Data on the prevalence and distribution of three most prominent genotypes in the cancer cases from different geographical regions of India shows a great regional variation [5-9, 15-17].

HPV 66, an intermediate risk genotype, had a prevalence of about $3 \%$ and a predominant type in cervicitis cases in the present study is rare in other population. The presence of HPV66 across all age groups and predominantly in cervicitis might indicate its possible role in the development of cervical cancer by triggering inflammation and persistent infection.

Low-risk HPV infection is a rare event in this population. Low-risk infection was observed in ICC cases only and always present in association with the high-risk genotypes. It remains unclear whether the association of low-risk genotypes with the high risk induces the progression of lesion or is it the effect of high-risk HPV that makes prone to the infection of other types.

The present study showed highest infection rate at the age of $\leq 35$ years among women without cancer. The age-specific trend of HPV infection among noncancer cases showed bimodal shaped infection peak which is similar to many other reports [28]. HPV infection at younger age reached its peak soon after sexual initiation [28].

Age specific multiple HPV genotype infection curve for ICC cases shows a bimodal peak. Highest peak is at $>55$ years and another shift is at $36-45$ years (Fig. 3). In women without cancer multiple infections were most frequently observed in the age group 3645 years and lowest in $>55$ years age group which corroborates with previous studies [25, 29]. Since sexual activity is higher in younger women, multiple genotype infection is higher at this age [30-32]. The higher frequency of multiple infections at the 36-45 years in both cancer and non cancer group might be due to multiple sexual partners at this age. The frequency of HPV infection and multiple types increases with age explains cumulative lifetime exposure [33], relative incompetency in viral clearance and insufficient adaptive immune responses at this age caused by hormonal changes at menopausal transition, contributing to HPV persistence or reactivation of latent HPV infections $[34,35]$.

The present study showed a significant association of multiple infections with increased tumor size. However, no significant association of multiple infections with other clinicopathological variables (age, stage, lymph node metastasis and histopathology) was found in the present work which agrees with the findings of Mungala et al [36]. Prognosis of cervical carcinoma is related to clinical stage, lymph node metastasis, parametrial invasion, primary tumor size, histological type, depth of cervical stromal invasion and lymph vascular space involvement [37]. Bachtiary et al. [38], in a study of 106 cervical cancer patients receiving radiation therapy, found that multiple-type HPV was an independent poor prognostic factor. Though there are several reports showing the association of multiple infections with cervical cancer $[39,40]$ the biological effect of multiple infections on cervical diseases has not yet been established. Synergistic effect of multiple infections by HPV genotypes on carcinogenesis is supported by many studies [7-9, 17, 26], while contradicting studies [25, 29, 31, 32, 35] invoke confusion. Association of multiple infections with tumor size is an interesting finding in this regard. Analysis on the correlation of tumor size with lymph node metastasis is under consideration which would throw more light on this. 
The rate of single genotype infection especially HPV 16 , was very high in the abnormal histopathological group as compared to the normal group which is in accordance with Baloch et al [28]. Hence, even without the information of the other genotypes, attention should be paid to those infected with single HPV16. Cervical cancer screening could be improved by detection of HPV16 besides cytology.

People staying in a rural area had 20-fold higher risk of acquiring HPV infection. Rural residential area reflects poor socio-economic condition possibly related to lack of access to proper care which facilitates infection and persistence of HPV and an increasing risk of cancer development [41]. In rural Odisha where sexual conception and behavior of women are conservative, men possessing multiple sexual partners might be a major source of HPV infection in a female. People from rural Odisha goes to the cities of neighboring states for their livelihood which provides an opportunity for having multiple partners. Our finding supports close surveillance of elderly women with HR-HPV infection and women of the rural area.

Approximately, more than $70 \%$ worldwide ICC could be prevented by the available bivalent HPV vaccinesCervarixTM (GlaxoSmithKline Biologicals, Rixensart, Belgium) and quadrivalent $\mathrm{HPV}$ vaccine- Gardasil ${ }^{\circ}$ (4vHPV) (Merck \& Co., Inc., Kenilworth, NJ, USA) $[26,27]$. The recently approved 9-valent vaccine covers HPV16/18/31/33/45/52/58 genotypes [42, 43].

To know the impact of a vaccine, estimating the attribution of cases to HPV types is confusing due to multiple genotypes present in the same tumor. In the present study high and low estimate of the impact of vaccines has been estimated to overcome this difficulty. Since the true potential impact lies somewhere between the high and low estimate [14] the average value of the two was considered for convenience. The present study shows a potential vaccine impact of $2 \mathrm{v}$ and $4 \mathrm{v}$ vaccines were 89.99 and $91.65 \%$ respectively. The impact would increase up to $92 \%$ with introducing 9v vaccine (Fig 4). It is similar to a study in French population [14]. The potential impact of the nonavalent vaccine in Asia and worldwide are 91.5 and $89.5 \%$ respectively [44]. Inconsistent with other studies, our findings also show an increase in the efficacy of vaccine with the switch from bivalent or tetravalent to the nonavalent vaccine [14, 45]. The result shows, for a maximum effectiveness of vaccination program, a $9 \mathrm{v}$ vaccine would be more useful.

HPV51, the third most prevalent genotype, and HPV66 another more frequent genotype of this region can't be cross protected even by the new $9 \mathrm{v}$ vaccine for being genetically unrelated. The risk of development of invasive cervical carcinoma associated with these genotypes needs to be estimated.

We were unable to assess HIV status or another disease for our participants, which could strongly influence the prevalence of HPV infection.

\section{Conclusions}

The present study provides first information about the genotype distribution among women with cervical cancer and without cancer cases of the state of Odisha which would help planning an appropriate strategy for disease monitoring. This study also offers the baseline data for future research and postvaccination surveillance in the region. The nonavalent vaccine would be the most effective vaccine to prevent cervical carcinoma in Odisha. Hence an effective vaccination program based on regional HPV epidemiological profile along with the cervical cancer screening is necessary to reduce the cervical cancer burden in India. Besides, HPV surveillance targeting elderly women and rural people will be useful.

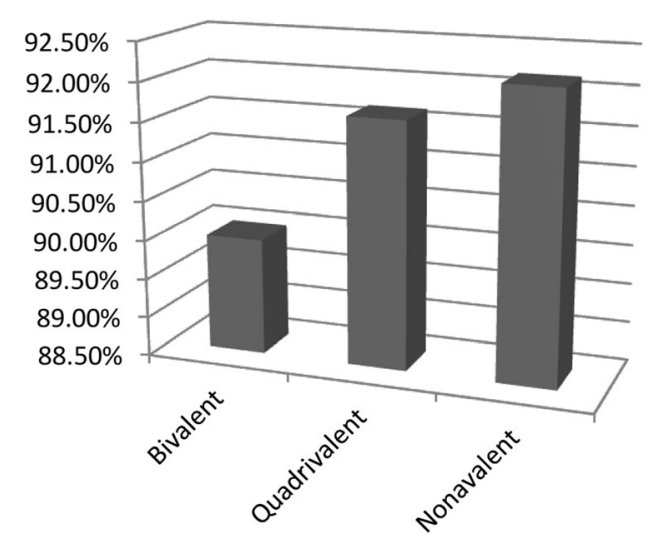

$\square$ Protected by vaccine

Fig. 4 Potential Impact of different HPV vaccines to prevent cervical cancer in Odisha 


\section{Additional file}

Additional file 1: Table S1. Distribution of HPV genotypes $(n=346)$ Table S3. Distribution of Low risk and high risk genotypes in different cytology. Table S4. HPV infection in different age group. Table S5. Clinico-Pathological characteristics in ICC patients and their association with multiple genotypes. (DOCX $22 \mathrm{~kb}$ )

\section{Acknowledgement}

We sincerely thank the Doctors and staffs of Department of gynecology Oncology, AHHRC and Department of gynecology, SCBMH, Cuttack, Odisha, India for access and help on sample collection. Author RS is grateful to ICMR for providing fellowship during the research work.

\section{Funding}

Author RS is the recipient of ICMR senior research fellowship, India (Award no-80/780/2012-ECD-I).

\section{Availability of data and materials}

Table S2 of additional file is not available to the readers as it will be used for future research.

\section{Authors' contribution}

1. RS- Conceived and designed the study, enrolled subject and collected samples, performed laboratory experiment, performed interpretation and analysis of data, drafted the manuscript. 2. BN- enrolled subjects, clinical examination of the subject, participated in sample collection 3. SKK-Participated in the interpretation of data,/ critically revised the manuscript 4. BD-participated in study design, Participated in interpretations and analysis of data,/critically revised the manuscript. All authors read and approved the final manuscript.

\section{Competing interest}

The authors' declare that they have no competing interests.

\section{Consent for publication}

Not applicable.

\section{Ethics approval and consent to participate}

The study proposal was reviewed and approved by the ethics committee of Regional medical research center (ICMR), Bhubaneswar, Odisha, India. The purpose and procedures of the study were explained to all the participants and informed written consent was obtained.

\section{Author details}

${ }^{1}$ Viral Research and Diagnostic Laboratory, Regional Medical research Centre (ICMR), Nalcosquare, Chandrasekharpur, Bhubanewar 751023, Odisha, India. ${ }^{2}$ Department of Gynecologic oncology, Acharya Harihara Regional Cancer Centre, Cuttack, Odisha, India. ${ }^{3}$ Institute of Medical Science and Sum Hospital, Shiksha O Anusandhana University, Bhubanewar, Odisha, India.

Received: 9 September 2016 Accepted: 17 December 2016 Published online: 05 January 2017

\section{References}

1. Globacan. Cervical cancer: estimated incidence mortality and prevalence worldwide in 2012. 2012; http://globocan.iarc.fr/old/FactSheets/cancers/ cervix-new.asp. Accessed 5 Dec 2015

2. Trottier $\mathrm{H}$, Burchell AN. Epidemiology of mucosal human papillomavirus infection and associated diseases. Public Health Genomics. 2009;12:291-307.

3. Bouvard V, Baan R, Straif K, Grosse Y, Secretan B, et al. A review of human carcinogens-Part B: biological agents. Lancet Oncol. 2009;10:321-2.

4. Herrero R. Human papillomavirus (HPV) vaccines: limited cross-protection against additional HPV types. J Infect Dis. 2009;199:919-22.

5. Sreedevi A, Javed R, Dinesh A. Epidemiology of cervical cancer with special focus on India. Int J Womens Health. 2015;7:405-14.

6. Bhatla N, Dar L, Patro AR, et al. Human Papillomavirus-Type Distribution in Women With and Without Cervical Neoplasia in North India. Int J Gynecol Pathol. 2008;27(3):426-30.
7. Basu P, Roychowdhury S, Bafna UD, Chaudhury S, et al. Human papillomavirus genotype distribution in cervical cancer in India: Results from a multi-center study. AsianPac J Cancer Prev. 2009;10:27-34.

8. Deodhar K, Gheit T, Vaccarella S, Romao CC, et al. Prevalence of Human Papillomavirus Types in Cervical Lesions from Women in Rural Western India. J Med Virol. 2012;84:1054-60.

9. Srivastava S, Shahi UP, Dibya A, Gupta S, Roy JK. Distribution of HPV Genotypes and Involvement of risk factors in cervical lesions and invasive cervical cancer: a study in an indian population. Int J Mol Cell Med. 2014;3:2.

10. Nayar R, Wilbur DC. The Pap test and Bethesda 2014. Cancer Cytopathol. 2015;123(5):271-81.

11. Sanger F, Nicklen $S$, Coulson AR. DNA sequencing with chain-terminating inhibitors. Proc Natl Acad Sci U S A. 1977:74:5463-7.

12. Sotlar K, Diemer D, Dethleffs A, Hack A, et al. Detection and Typing of Human Papillomavirus by E6 Nested Multiplex PCR. J Clin Microbiol. 2004;42:3176-84.

13. MediCal version 14.10.2(7) http://www.medcalc.org/index.php. Accessed 17 Dec 2015.

14. Riethmuller D, Jacquard A, Guily JL, Aubin F. Carcopino X et al Potential impact of a nonavalent HPV vaccine on the occurrence of HPV-related diseases in France. BMC Public Health. 2015;15:453.

15. Sauvaget $C$, Nene $B$, Jayant $K$, et al. Prevalence and determinants of highrisk human papillomavirus infection in middle-aged Indian women. Sex Transm Dis. 2011;38(10):902-6.

16. Sowjanya AP, Jain M, Poli UR, et al. Prevalence and distribution of high-risk human papillomavirus (HPV) types in invasive squamous cell carcinoma of the cervix and in normal women in Andhra Pradesh. India BMC Infect Dis. 2005:5:116.

17. Datta P, Bhatla N, Pandey RM, et al. Type-specific incidence and persistence of HPV infection among young women: a prospective study in North India. Asian Pac J Cancer Prev. 2012;13(3):1019-24

18. Pillai RM, Babu JM, Jissa VT, Lakshmi S, Chiplunkar SV, et al. Region-wise distribution of high-risk human papillomavirus types in squamous cell carcinomas of the cervix in India. Int J Gynecol Cancer. 2010;20:1046-51.

19. Bhatla N, Dar L, Patro AR, Kriplani A, Gulati A, Verma K, Broor S, Shah KV, Gravitt PE. Human papillomavirus type distribution in cervical cancer in Delhi, India. Int J Gynecol Pathol. 2006;25(4):398-402.

20. Munjal K, Adamson C, Rajendran V, Nandedkar S. Human papillomavirus type distribution in invasive cervical cancers from Madhya Pradesh: implications for vaccination programs in central India. Int J Gynecol Pathol. 2014;5:531-6.

21. Gul S, Murad S, Javed A. Prevalence of High risk Human Papillomavirus in cervical dysplasia and cancer samples from twin cities in Pakistan. Int J Infect Dis. 2015:34:14-9.

22. Siddiqa A, Zainab M, Qadri I, Bhatti MF, Parish JL. Prevalence and genotyping of high risk human papillomavirus in cervical cancer samples from Punjab, Pakistan. Viruses. 2014;6:2762-77.

23. Raza SA, Franceschi S, Pallardy S, Malik FR, Avan BI, Zafar A, Pervez S, Serajuddaula S, Snijders PJF, van Kemenade FJ, et al. Human papillomavirus infection in women with and without cervical cancer in Karachi, Pakistan. Br J Cancer. 2010:102:1657-60.

24. Khan S, Jaffer NN, Khan MN, Rai MA, Shafiq M, Ali A, Pervez S, Khan N, Aziz A, Ali SH. Human papillomavirus subtype 16 is common in Pakistani women with cervical carcinoma. Int J Infect Dis. 2007;11:313-7.

25. Chen Q, Xie L-X, Qing Z-R, Li L-J, Luo Z-Y, et al. Epidemiologic Characterization of Human Papillomavirus Infection in Rural Chaozhou, Eastern Guangdong Province of China. PLoS ONE. 2012;7(2):e32149. doi:10.1371/journal.pone.0032149.

26. Sanjosé SD, Diaz M, Castellsagué $X$, et al. Worldwide prevalence and genotype distribution of cervical human papillomavirus DNA in women with normal cytology: a meta-analysis. Lancet Infect Dis. 2007:7(7):453-9.

27. Guan P, Howell-Jones R, Li N, Bruni L, et al. Human papillomavirus types in 115,789 HPV-positive women: A meta-analysis from cervical infection to cancer Int. J Cancer. 2012;131:2349-59.

28. Baloch Z, Li Y, Yuan T, Feng Y, et al. Epidemiologic characterization of human papillomavirus (HPV) infection in various regions of Yunnan Province of China. BMC Infect Dis. 2016;16:228

29. Resende LS, Rabelo-Santos SH, Sarian LO, Alves RR, Ribeiro AA, et al. A portrait of single and multiple HPV type infections in Brazilian women of different age strata with squamous or glandular cervical lesions. BMC Infect Dis. $2014 ; 14: 214$ 
30. Gonzalez-Bosquet E, Esteva C, Munoz-Almagro C, Ferrer P, Perez M, Lailla $J \mathrm{M}$. Identification of vaccine human papillomavirus genotypes in squamous intraepithelial lesions (CIN2-3). Gynecol Oncol. 2008;111(1):9-12.

31. Sjoeborg KD, Trope A, Lie AK, Jonassen CM, Steinbakk M, Hansen M, et al. HPV genotype distribution according to the severity of cervical neoplasia. Gynecol Oncol. 2010;118(1):29-34.

32. Martins TR, Oliveira CM, Rosa LR, Centrone CC, et al. HPV genotype distribution in Brazilian women with and without cervical lesions: correlation to cytological data. Virol J. 2016;13:138.

33. Lin $M$, Yang $L Y, L i L, W u$ JR, Peng YP, et al. Genital human papillomavirus screening by gene chip in Chinese women of Guangdong province. Aust N Z J Obstet Gynaecol. 2008;48:189-94.

34. Herrero R, Castle PE, Schiffman M, Bratti MC, Hildesheim A, Morales J, et al. Epidemiologic profile of type-specific human papillomavirus infection and cervical neoplasia in Guanacaste. Costa Rica J Infect Dis. 2005:191:1796-807.

35. Hildesheim A, Wang SS. Host and viral genetics and risk of cervical cancer: a review. Virus Res. 2002;89:229-40.

36. Munagala R, Donà MG, Rai SN, Jenson AB, Bala N, Ghim SJ, et al. Significance of multiple HPV infection in cervical cancer patients and its impact on treatment response. Int J Oncol. 2009;34:263-71.

37. Kosary CL. FIGO stage, histology, histologic grade, age and race as prognostic factors in determining survival for cancers of the female gynecological system: an analysis of 1973-87 SEER cases of cancers of the endometrium, cervix, ovary, vulva, and vagina. Semin Surg Oncol. 1994;10(1):31-46.

38. Bachtiary B, Obermair A, Dreier B, Birner P, Breitenecker G, Knocke TH, et al. Impact of multiple HPV infection on response to treatment and survival in patients receiving radical radiotherapy for cervical cancer. Int J Cancer. 2002;102:237-43.

39. Chagas BS, Comar M, Gurgel APAD, Paiva S, Seraceni S, de Freitas AC, et al. Association Study between Cervical Lesions and Single or Multiple Vaccine-Target and Non-Vaccine Target Human Papillomavirus (HPV) Types in Women from Northeastern Brazil. PLoS ONE. 2015;10(7): e0132570. doi:10.1371/journal.pone.0132570.

40. Chaturvedi AK, Katki HA, Hildesheim A, Rodríguez AC, Quint W, Schiffman $M$, et al. Human papillomavirus infection with multiple types: pattern of coinfection and risk of cervical disease. J Infect Dis. 2011;203:910-20.

41. Mitchell S, Sekikubo M, Biryabarema C, Byamugisha J, Steinberg M, Jeronimo J, et al. Factors associated with high-risk HPV positivity in a low-resource setting in sub-Saharan Africa. Am J Obstet Gynecol. 2014;210(1):81.e1-7.

42. Press Announcements-FDA approves Gardasil 9 for Prevention of Certain Cancers Caused by Five Additional Types of HPV. Available online: http:// www.fda.gov/newsevents/newsroom/pressannouncements/ucm426485. htm. Accessed 15 Feb 2016).

43. European Medicines Agency_News and Events_-Gardasil 9 Offers Wider Protection against Cancers Caused by Human Papillomavirus (HPV). Available online: http://www.ema.europa.eu/ema/index.jsp?curl=pages/news_and events/news/2015/03/news_detail_002295.jsp\&mid=WC0b01ac058004d5c1. Accessed 15 Feb 2016

44. Loya A, Serrano B, Rasheed F, Tous S, et al. Human Papillomavirus Genotype Distribution in Invasive Cervical Cancer in Pakistan. Cancers. 2016:8:72.

45. Van de Velde N, Boily MC, Drolet M, Franco EL, Mayrand MH, Kliewer EV, et al. Population-level impact of the bivalent, quadrivalent, and nonavalent human papillomavirus vaccines: a model-based analysis. J Natl Cancer Inst. 2012;104(22):1712-23.

\section{Submit your next manuscript to BioMed Central and we will help you at every step:}

- We accept pre-submission inquiries

- Our selector tool helps you to find the most relevant journal

- We provide round the clock customer support

- Convenient online submission

- Thorough peer review

- Inclusion in PubMed and all major indexing services

- Maximum visibility for your research

Submit your manuscript at www.biomedcentral.com/submit

C) Biomed Central 